International Journal of Engineering \& Technology, $7(3.34)(2018) 82-85$
SPC
Website: $w$ ww.sciencepubco.com/index.php/IJET
Research paper

\title{
HPV Sensing by CNTFET Array Nanobiosensor.
}

\author{
Santhosh Kumar D. R ${ }^{1 *}$, Dr P.V. Rao ${ }^{2}$ \\ ${ }^{1}$ Jain University, Research Scholar, Department of Electronics, Bengaluru \\ ${ }^{2}$ Vignana Bharati Institutes of Technology, Professor, Department of Electronics, Hyderabad. \\ *Corresponding authorE-mail:drsan6@gmail.com
}

\begin{abstract}
Women life is threatened by the cervical cancer each time worldwide. The cervical cancer high grade pre cancer is due to HPV E6 and E7 agents. Nano materials show a key role in medical analysis and action. CNTs (Carbon Nano Tube) have very unique electrical and mechanical properties which are useful in the bio-application. The conventional based biosensors can be improved by CNT based biosensors with respect to sensitivity, selectivity and simple in operation. In comparison with the silicon transistors, CNTFET (Carbon Nano Tube Field Effect Transistor) nano device takes less power and performs faster. The research paper covers working of CNTFET based nano biosensor to detect cervical cancer antibody. 4 x 4 CNTFET sensor array is designed to detect antibody variations on CNTFET gate. The sensor current varied from $4.286 \mu \mathrm{A}$ to $15.435 \mu \mathrm{A}$ for gate voltage varied from $0.2 \mathrm{~V}$ to $1.06 \mathrm{~V}$. The improved $64 \mathrm{CNTFET}$ based biosensor performs better in sensing the analyte of different concentrations.
\end{abstract}

Keywords: cancer, CNTFET, HPV, nano electronics, sensor array

\section{Introduction}

In worldwide the major cause of adult deaths is cancer. The main reason for women deaths in both rural and urban areas is cervical cancer [1]. According to the WHO it is the most common cancer among women. By 2030 it is projected to kill about 474,000 women every year [2]. In sub Saharan Africa, India and other area of the developing world it is the main cancer among women which is both avoidable and treatable [3]. It grows slowly and headed by precancerous alterations in the cervix. Precancerous alterations in the cervix are histologically determined as cervical intraepithelial neoplasia (CIN) similarly is known at wavering stages of severity: CIN1, CIN2, and CIN3. The last comprises CIS (carcinoma in situ) [4].

Invasive cervical cancer (ICC) is sluggish from the development of neoplasia. The cervical cancer due to HPV infection follows different stages like transmission, infection, persistent infection showing precancerous changes and ICC. Diffusion of HPV to the anogenital area occurs mainly due to consequence of skin-to-skin or mucosa-to-mucosa interaction. The change in malicious HPV affected cells is established to be found by the combination of viral DNA to host genome. The virus replicates precisely in maximum at low-grade cuts [4]. The reason for virtually every cervical cancer is known to be infection through oncogenic HPV varieties. The $12 \mathrm{HPV}$ varieties intensely linked with cervical cancer are 16, $18,33,31,39,35,45,52,51,58,56$, and 59. Additional probable carcinogenic HPV varieties comprise 82, 53, 67, 66, 68, 73, 70, and 26. The Eight HPV varieties $(18,16,33,31,35,52,58$, and 45) are the reason for 95 in each hundreds of SCCs progressive for HPV deoxyribonucleic acid (DNA). The HPV varieties 16 and 18 alone are accountable for roughly 70 in each hundreds of cervical cancer circumstances [4].

The cervical cancer selection methods are cervical smear (pap), fluid sampling methods by automating thin layer preparation, automated cervical screening, Visual inspection of cervix after applying Lugol's iodine (VILI) or acetic acid (VIA), Neuro medical schemes, Polar probe, Laser induced fluorescence, Speculoscopy, Cervicography, HPV testing. At present, cervical cytology is widely noticed as the important standard for cervical cancer selection in absolutely progressive nations. So it is necessary to make low cost screening approaches for cervical cancer [5].

Biomarkers are particles that can be precisely identified and assessed as pointers of usual or disease practices and pharmacologic reactions to recovery [6]. Proper selection of biomarkers is important for diagnostic purpose. It is a necessity to detect a disease through biomarkers intensities. Biomarkers are quantifiable and assessable biological factors such as macromolecule volatile compounds, concentration, and deviation in genes, originate in the company of organic material, help as pointers for strength and bodily processes linked estimates. The choice of biomarkers is so important for disease observation at the earliest before they establish [7]. Different varieties of biomarkers need to be tested for disease analysis. These biomarkers are made either one by the tumour itself or else by the physique in reaction to the occurrence of cancer. The two groups of biomarker applications are viral and cellular. Specified restrictions in the usage of equally cytology as well as HPV DNA focused methods as separate examinations for screening; the emphasis for cervical cancer suppression study is on development mode also verification of innovative sicknessprecise biomarkers of HPV-related transformation. Development from a temporary to a varying infection of HPV is measured by a robust rise of protein and HPV E6/E7 mRNA presence. The biomarker broadly assessed is p16ink4a, a cyclindependent kinase inhibitor that exists decidedly over conveyed in cancerous plus cervical precancerous tissue [8].

Biosensors are proficient for sensing precise biological analytes as well as varying their existence or concentration into definite, thermal, electrical, optical or else additional signal for analysis. In 1962, Clark and Lyons designed first biosensor for glucose measurement. The International Union of Pure and Applied Chemistry 
expresses a biosensor as, a device that practices biochemical reactions arbitrated by isolated organelles, enzymes or else whole cells to sense the effects of chemical compounds by thermal, optical or electrical signals. Biosensors can be categorized into four classes on the foundation of the kind of transducer used: optical (interferometric, colorimetric, luminescent, and fluorescent) biosensors, mass-grounded piezoelectric as well as acoustic wave biosensors, calorimetric, plus electrochemical (potentiometric, amperometric, and conductometric) biosensors [8].

\section{Biosensor and CNTFET Properties}

The structure of CNTFET is shown in figure (1), contains top, channel and bottom and formed from nanotube that links the source plus drain. The conduction properties of the nanotube are changed by the voltage applied on the two gates, and it changes the flow of current amongst source plus drain. The flow of current is directly proportionate to the applied gate voltage of the CNTFET.

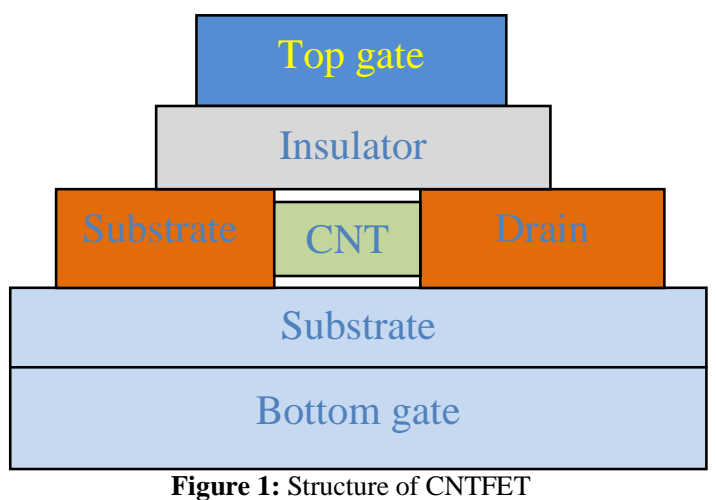

The CNT type like single (SWCNT) and multi-wall (MWCNT) are turned with carbon nanoribbon. The ninithi simulation software is used to show the CNT structure with $m=19, n=0$ and length=32 in figure (2). The CNT valence and electron band is shown in figure (3). A SWCNT is involved of a single cylinder. A MWCNT is collected of more than one cylinder. The SWCNT might be semiconductor or else conductor, actuality depending upon its chirality vector. The chirality vectors are determined by $(\mathrm{n} 1, \mathrm{n} 2)$ indices, which are the wrapping vector that the graphite sheet is turned up [9].

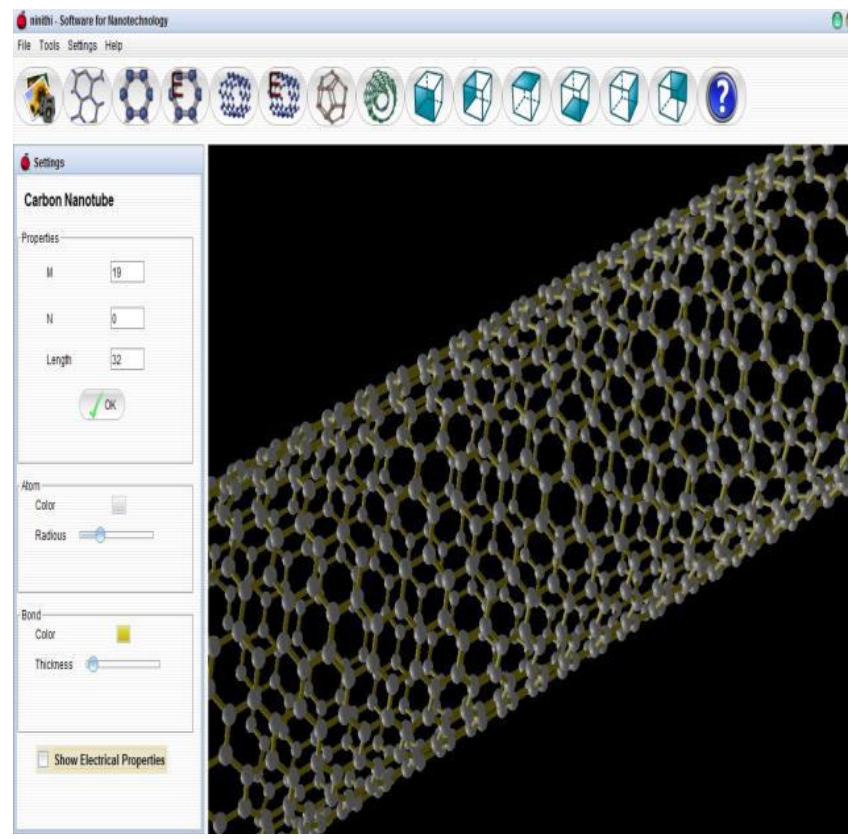

Figure 2: CNT structure with chirality $(19,0)$

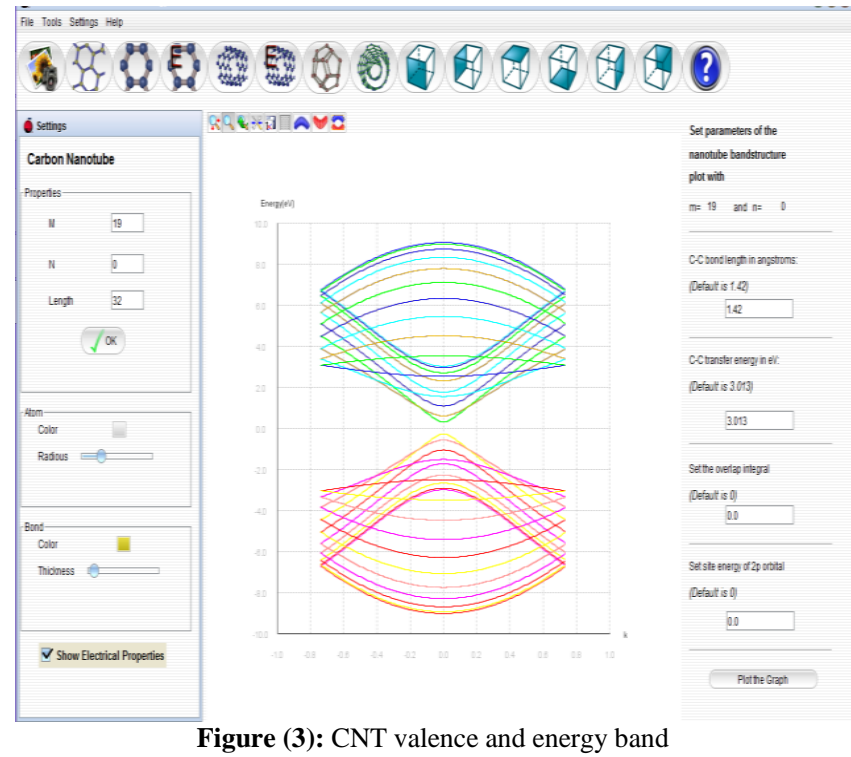

The transconductance gain is indicated by gm1 also by the output resistance which is denoted through rds1 and is given in Equations (1) and (2).

$g m_{1}=\sqrt{2 \mu_{n} C_{o x}\left(\frac{W}{L}\right)} I_{\text {bias }}$

$r_{d s 1}=\frac{A^{t} L(u m)}{I_{D}}$

The $\mathrm{I}_{\text {semi }}$ current and $\mathrm{I}_{\mathrm{BT}}$ current establish the main current flow in CNTFET. The chirality vectors are used as control parameters for channel diameter design as well as similarly direct the maximum current flow inside CNTFET. The CNT diameter is Dent, which is given by Equation (3).

Dent $=\frac{a \sqrt{n_{1+}^{2} n_{2+}^{2} n_{1} n_{2}}}{\pi}=0.0783 \sqrt{n_{1+}^{2} n_{2+}^{2} n_{1} n_{2}}$

In the Equation (3) the chirality vectors are $\mathrm{n}_{1}$ and $\mathrm{n}_{2}$ besides a $=2.49$ angstrom unit. The CNTFET current from drain to source is known by Equation (4).

$$
I_{C N T F E T}=n g_{C N T}\left(V_{D D}-V_{S S}-V_{i n C N T}\right)
$$

The $32 \mathrm{~nm}$ CNTFET electrical properties of are quicker and capable of handling greater currents in the range of $70 \mu \mathrm{A}$. Grounded on the considerations offered in [10] for biosensor design, in this effort, an innovative architecture is considered using CNTFET.

The integration of DNA with nanobiosensor is designed to detect HPV. The preparation of nanobiosensor is done by alteration of Amino-silane. 3-Aminopropyltriethoxysilane (APTES) joined through $\mathrm{NH}_{2}$ is altered into APTES-COOH by means of Succinic Anhydride. The probe of HPV DNA is connected towards $\mathrm{TiO}_{2}$ by submerging the $\mathrm{TiO}_{2}$ in APTES as well as it is retained to dry for further usage as a probe. Subsequently, probe fixing into $\mathrm{TiO}_{2}$, the hybridization is conceded by washing as well as denaturation to remove the redundant samples of HPV DNA after the $\mathrm{TiO}_{2}$ probe. An even layer is set by an auxiliary handling of silicon surface through APTES. Immobilization of APTES-COOH taking place on thin $\mathrm{TiO}_{2}$ film displayed advance in flow of current changing from $0.21 \mu \mathrm{A}$ near to $0.81 \mu \mathrm{A}$ for an applied bias potential of $0.2 \mathrm{~V}$ $-1 \mathrm{~V}$. The flow of current in the experiment [10] exhibited that for an applied bias voltage of $0.8 \mathrm{~V}$, the variation in current is of 0.6 $\mu \mathrm{A}$. In the direction to increase the HPV sensing activity, it is 
essential to substitute the $\mathrm{TiO}_{2}$ by carbon nanotube. In the current research effort, carbon nanotube transistor is applied to attain additional amplification and increase the current rating.

The CNTFET device is submerged in cervical specific antibody (anti-CSA) solution approximately greater than 12 hour at $4^{\circ} \mathrm{C}$. Due to this anti-CSA is associated to the group of aldehyde by forming a CNTFET biosensor [11]. The fabrication as well as operation of CNTFET device is carried out in the n-channel depletion characteristics. The groups of aldehyde after glutarldehye linked to the APTES amino groups stand connected to the surface of the biosensor for immobilizing the anti CSA particles. The biosensor for sensing HPV cells is employed with CNTFET for increasing the biosensor current sensitivity. The CNTFET possess larger ON current. Due to the smaller structure of molecules the CNTFET device, future size scaling, away from anything presently obtainable progressive lithographic approaches permit, is achievable. The benefit of CNTFET device lies in the threshold voltage which can be found through a suitable diameter for its CNTs [12]. CNTFET can be rearranged in the digital circuits besides it is suitable in the design of multi- $\mathrm{V}_{\text {th }}$ circuits [13].

The $n$ FET $\mathrm{V}_{\text {th }}$ is about $0.25 \mathrm{~V}$ also for pFET $\mathrm{V}_{\text {th }}$ is $-0.27 \mathrm{~V}$. By the application of Vgs input voltage the ON current grows linearly. The high channel mobility is offered by the CNT to automatically raise current further linearly. By increasing the number of tubes the CNTFET current is increased. The $32 \mathrm{~nm}$ CNTFET offers faster and high current in the range of $70 \mu \mathrm{A}$. Established on the findings existing in [10] for the biosensor design, in this research activity, an innovative design is employed using CNTFET. In the reference research activity an arrays sensor network and control unit to detect cancer is discussed in [14]. The testing sample is positioned above the arrays of sensor, when the arrays surface is spread over by the analyte, every single biosensor arrests the antigen existing in the analyte also electro potential is formed at every node as well as the resultant current flows in the sensor. After placing the analyte on the array, the array sensor nodes react to the analyte where its concentration is more. In order to get better response and improvisation in the sensing activity an improvised sensor array is designed.

\section{Improved Sensor Array Design}

Previous reference work like sensor array design for prostate cancer detection has used the dgfet 64 array to sense the analyte [15] In one more research Prostate Specific Antigen contained in the sample of blood is sensed by DNA strands, where three diverse DNA strands are assessed for their performance [16]. The CSA marker existing in the solution reacts with the anti-CSA molecule on the CNTFET channel to change the gate voltage, thus regulating the current to flow through the channel measured at the drain end. Higher concentration of CSA agent in the solution increases the drain current and hence CNTFET sensor readings are calibrated for detecting cervical cancer [17].

The present work is on using carbon nanotube instead of $\mathrm{TiO}_{2}$ to better the amplification process. CNTs place an important role in nano electronics because of its special electrical properties. It acts as metal or semiconductor [18]. Because of nano meter diameter range CNT's possess larger surface area. A CNT is joined to two metal electrodes which is the reason behind its field effect transistor characteristic. CNT conduction can be changed by applying a gate potential [19]. The backscattering is suppressed by CNTFET unique one dimension band structure. It is faster and consumes less power than silicon transistor, which makes it ideal device for high frequency and lower power applications [12].

The biopotential of equivalent which is generated with chemical response of antigen with antibody is referred from [21,10] as well as it is used in the improved 64 CNTFET sensor array and is simulated using Hspice. The biosensor response is shown in figure 4. In the first arrangement a 4 x 4 CNTFET array is designed to sense the analyte. For the equivalent electro potential applied the 4 $\mathrm{x} 4$ CNTFET array gives better result series 2 in comparison with the series 1 reference work [10]. The current values varies from $4.286 \mu \mathrm{A}$ to $15.435 \mu \mathrm{A}$ for gate voltage varied from $0.2 \mathrm{~V}$ to 1.06 $\mathrm{V}$ as shown in figure 4 . In order to enhance the sensing activity of the sensor to detect analyte, the improved array of CNTFET is designed. For different analyte concentration applied as equivalent electro potential the improved 64 CNTFET array sensor response is better in comparison with the reference work [10] result. The presence of analyte in the sample may vary with respect to cell area. The increase in Id $(\mu \mathrm{A})$ is an indication of presence of analyte in the sample. The CNTFET sensor array when placed with the sample to detect analyte, any one sensor may detect the analyte. The high current is the indication of the anlyte present in the sample. So, the variations shown in the figure 4 , series 3 are due to the different sensor detecting the anlyte at various places in the sample. These variations are indicated in the graph with increased value in the $\mathrm{Id}(\mu \mathrm{A})$ obtained from the sensor. The improved CNTFET sensor array is better in detecting the analyte of interest.

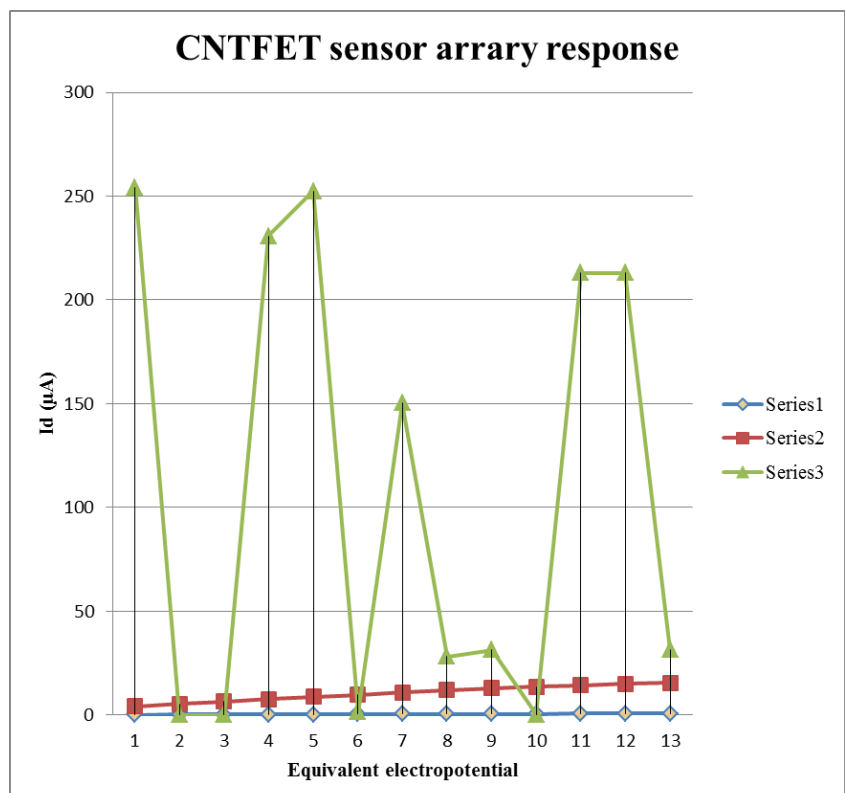

Figure 4: The graph shows the current obtained for various equivalent electropotential applied to the improved 64 CNTFET sensor array.

Series 1 represents the reference work [10].

Series 2 represents the $4 \times 4$ CNTFET sensor.

Series 3 represents the present work for improved $64 \mathrm{CNT}$ FET sensor.

\section{Conclusion}

The application of CNTFET based nano biosensor to detect cervical cancer is better in result compared to the reference work [10]. The analyte of interest is sensed by the CNTFET nano biosensor through variations in the current because of different analyte concentration. The $4 \times 4$ array current varies from from $4.286 \mu \mathrm{A}$ to $15.435 \mu \mathrm{A}$ for gate voltage varied from $0.2 \mathrm{~V}$ to $1.06 \mathrm{~V}$. To improve the $4 \times 4$ CNTFET array sensing, the 64 CNTFET array performs better in detecting the analyte. The different current variations of higher values indicate the presence of analyte shown in series 3 of figure 4 . The application of CNTFET is vital in the early detection of cervical cancer.

\section{References}

[1] Dikshit, Prakash C Gupta et al., for the Million Death Study Collaborators. Cancer mortality in India: a nationally representative 
survey.www.thelancet.com Published online March 28, 2012; DOI: 10.1016/S0140-6736(12)60358-4.

[2] Justin O. Parkhurst \& MadhulikaVulimiri (2013) Cervical cancer and the global health agenda: Insights from multiple policy-analysis frameworks, Global Public Health: An International Journal for Research, Policy and Practice, 8:10, 10931108,DOI:10.1080/17441692.2013.850524. http://dx.doi.org/10.1080/17441692.2013.850524.

[3] Chankapa YD, Pal R, Tsering D, Correlates of cervical cancer screening among underserved women. Indian Journal of Cancer January-March 2011 |Volume 48 | Issue 1. 10.4103/0019509X.75823.

[4] Vesco KK et al, Screening for Cervical Cancer: A Systematic Evidence Review for the U.S. Preventive Services Task Force. Evidence Synthesis No. 86. AHRQ Publication No. 11-05156-EF-1. Rockville, MD: Agency for Healthcare Research and Quality; May 2011.

[5] Neerja Bhatia et al., Evaluation of adjunctive tests for cervical cancer screening in low resource settings. Indian Journal of Cancer, April-June 2007, Volume 44, Issue 2.

[6] Gouvêa CMCP (2011) Biosensors for health applications. Universidade.

[7] Ngoepe M, Choonara YE, Tyagi C, Tomar LK, du Toit LC, Kumar,P, Ndesendo VMK, Pillay V (2013) Integration of biosensors, and drug delivery technologies for early detection and chronic management of illness. Sensors 13:7680-7713. doi:10.3390/s130607680.

[8] Fracchiolla NS, Artuso S, Cortelezzi A (2013) Biosensors in clinical practice: focus on oncohematology. Sensors 13:64236447.doi:10.3390/s130506423.

[9] Mohammad Hossein Moaiyeri, Reza Faghih Mirzaee, Keivan Navi and Omid Hashemipour, -Efficient CNTFET-based Ternary Full Adder Cells for Nanoelectronicsl, Nano-Micro Lett.3(1), 43-50 (2011). http://dx.doi.org/10.3786/nml.v3i1.p43-50

[10] N. Azizah, U.Hashim, Sh. Nadzirah, A. Rahim Ruslinda, Rapid and sensitive strategy for Human Papillomavirus (HPV) detection using a gene-based DNA nanobiosensor, in: Biomedical Engineering and Sciences (IECBES), 2014 IEEE Conference on, IEEE, 2014.

[11] J. Deng, H.-S.P. Wong, A compact SPICE model for carbonnanotube field-effect transistors including nonidealities and its application-Part I:Model of the intrinsic channel region, IEEE Trans. Electron Devices 54 (12) (2007) 3186-3194.

[12] S. Ashok Kumar, T. Shanmuganantham, Implantable CPW Fed Monopole H-slot Antenna for $2.45 \mathrm{GHz}$ ISM Band

[13] Applications, Int. J. Electron. Commun., Elsevier Publications

[14] 68 (7) (2014) 661-666.

[15] F.R. Madriz et al, Circuit modeling of high-frequency electrical conduction in carbon nanofibers, IEEE Trans. Electron Devices 56 (8) (2009) 1557-1561.

[16] S. Ushaa, M. Madhavilatha, G.M. Rao, Design and analysis of nanowire sensor array for prostate cancer detection, Int. J. Nano Biomater. 3 (3) (2011) 239-255.

[17] B.N. Shobha, N. J. R. Muniraj, Design, Modeling and Simulation of Prostate Cancer Biosensor with ssDNA biomarker and DGFET Biosensor (IJCSIT) International Journal of Computer Science and Information Technologies, Vol. 5 (2), 2014, 2612-2620.

[18] B. N. Shobha, N. J. R. Muniraj, Design, modeling and performance analysis of carbon nanotube with DNA strands as biosensor for prostate cancer, Microsyst Technol (2015) 21:791-800, DOI 10.1007/s00542-014-2281-x.

[19] B.N. Shobha, N.J.R. Muniraj, Modeling and simulation of cntfet for,prostate cancer detection, 30th November 2014. Vol. 69 No.3, ISSN: 1992-8645.

[20] Carbon Nanotubes: The Hub of Nanoelectronics, P.I. Okwu1 and I.N. Onyeje2, International Journal of Engineering Trends and Technology (IJETT) - Volume 4 Issue 10 - Oct 2013.

[21] Carbon Nanotubes - A Potential Material for Affinity Biosensors, Vepa K. Rao, S. Suresh, Mukesh K. Sharma, Ajay Gupta and R. Vijayaraghavan Defence Research \& Development Establishment, Gwalior, India.

[22] S. Wind et al, Vertical scaling of carbon nanotube field-effect transistors using top gate electrodes, Appl. Phys. Lett. 80 (20) (2002) 3817-3819.

[23] Z. Chen et al, The role of metal-nanotube contact in the performance of carbon nanotube field-effect transistors, Nano Lett. 5 (7) (2005) 1497-1502 